\title{
Progress in mechanics of soils and general granular flows: preface
}

\author{
Pasquale Giovine · Paolo Maria Mariano • Giuseppe Mortara • \\ Kenichi Soga
}

Published online: 20 February 2019

(C) Springer Nature B.V. 2019

Soils are combinations of particles with a wideranging size (from large blocks to gravel, sand, silt, clay) and degree of compaction. They can be dry or with voids partially or fully filled by fluids. Their structure shows pronounced anisotropy. Their mechanical behavior suggests a number of modeling possibilities and problems. Common schemes referring to elastic-plastic and elastic-viscous or viscoplastic behavior of materials in the traditional (Cauchy-type) format of continuum mechanics offer approximate (although often reliable) representations of soil behavior, which are useful to common engineering practice.

However, at times such descriptions appear limited or even insufficient in various critical circumstances such as, for example, those occurring under strong ground motion: liquefaction, fracturing, reorganization of texture, various types of instabilities. We can add all cases in which environmental conditions produce chemical reactions altering mechanical

P. Giovine $(\bowtie) \cdot$ G. Mortara

University Mediterranea of Reggio Calabria,

Reggio Calabria, Italy

e-mail: giovine@unirc.it

P. M. Mariano

University of Florence, Florence, Italy

K. Soga

University of California at Berkeley, Berkeley, USA properties, but also those cases in which the flow of fluids through the solid skeleton is not quasi-static. This implies rearrangement in at least part of the skeleton itself.

Physics suggests us to call upon and sometimes to refine the theory of mixtures or even essential concepts from the mechanics of complex materials, as soils are, at least to a certain extent. In the latter case we include geometric descriptors of material changes at low spatial scales. In fact, the construction of a model in continuum mechanics does not necessarily reduce to the mere choice of a constitutive structure. Rather, its formulation starts from the level of detail we adopt in describing the body morphology and its changes. The representation of interactions is consequent. Their balances emerge from requirements of external power invariance under changes in observers.

Grossly, we may subdivide modeling approaches into heuristic and 'rationally deduced' classes. The common boundary is not sharp, and it is rather a sort of extended mushy zone.

In heuristic class, we act mainly on the basis of data-fitting schemes or by analogy to well established matter. However, although analogy seems at times a reasonable way to act, above all when we fill having nothing else at disposal, the models obtained acting by analogy have, by construction, hardly controllable theoretical foundations. Hence, such approaches may imply (or may be based on) just a hope. For data-fitting schemes, we recall that data are always limited. Also, 
to get them, we need to design experiments; and the plan requires at least a rough preliminary theoretical view on the behavior we aim at describing.

Alternatively, we can rationally derive models from first principles motivated by recorded phenomenology or the one we imagine (i.e., the result of a Gedankenexperiment). Eventually, even in this case, after deriving the essential structures of a model, and proving rigorously what is available to our abilities, we need the introduction of parameter values, i.e., we require knowledge of experimental data.

Care is essential in managing and interpreting experiments. Of course, this is a general indication for any experimental champaign. However, it has to be particularly considered in experiments on soils. As regards this aspect, a remark by P. G. De Gennes applies:

"We fill a glass column with sand. This innocent statement hides many subtleties. Did we fill it from a jet of sand near the axis, or did we sprinkle the sand over the whole section? Did we shake the object after filling? (Rev. Modern Phys., 71, 1999, S374-S382.)

De Gennes mentions sand, but his remark applies to the whole granular matter, considered, in general, as a system of particles interacting just by collision, a cluster in which the size of granules is larger than one micron (if we include powders), in essence a singlephase body. Soils are not always so simple, rather they are commonly multi-phase and their components interact one another, increasing the level of modeling problems. However, even the description of granular matter mechanics proposes challenging questions, especially when we consider fast and large scale granular flows and aim at transiting from mass-pointtype or small-rigid-body-type descriptions of each granule to a continuum representation, which is necessary due to the plurality of grains and the prohibitive computational cost of multi-body views on wide granular clusters.

In summary, theoretical, experimental, and computational questions emerging in the analysis of soils are challenging, often very intricate, at times subtle.

Here, we offer a collection of results on the mechanics of soils, which indicate (or, at least, allow readers to perceive at least a portion of) the plurality of views, problems, and open perspectives in the field.

\section{Compliance with ethical standards}

Conflicts of interest The authors declare that they have no conflict of interest. 